\title{
Risk Adjustment for High Utilizers of Public Mental Health Care
}

\author{
Kanika Kapur $^{1 *}$ Alexander S. Young ${ }^{2,3}$ and Dennis Murata ${ }^{4}$ \\ ${ }^{1}$ RAND Corporation, 1700 Main Street, Santa Monica, CA 90401, USA ${ }^{2}$ West Los Angeles Veterans HealthCare Center, MIRECC, Building 210A, Los \\ Angeles, CA 90073, USA ${ }^{3}$ UCLA Neuropsychiatric Institute, 10920 Wilshire Boulevard, Suite 300, Los Angeles, CA 90024, USA ${ }^{4}$ Los Angeles County \\ Department of Mental Health, 550 South Vermont Avenue, 12th Floor, Los Angeles, CA 90020, USA
}

\begin{abstract}
Background: Publicly funded mental health systems are increasingly implementing managed care systems, such as capitation, to control costs. Capitated contracts may increase the risk for disenrollment or adverse outcomes among high cost clients with severe mental illness. Risk-adjusted payments to providers are likely to reduce providers' incentives to avoid or under-treat these people. However, most research has focused on Medicare and private populations, and risk adjustment for individuals who are publicly funded and severely mentally ill has received far less attention.

Aims of the Study: Risk adjustment models for this population can be used to improve contracting for mental health care. Our objective is to develop risk adjustment models for individuals with severe mental illness and assess their performance in predicting future costs. We apply the risk adjustment model to predict costs for the first year of a pilot capitation program for the severely mentally ill that was not risk adjusted. We assess whether risk adjustment could have reduced disenrollment from this program. Methods: This analysis uses longitudinal administrative data from the County of Los Angeles Department of Mental Health for the fiscal years 1991 to 1994. The sample consists of 1956 clients who have high costs and are severely mentally ill. We estimate several modified two part models of 1993 cost that use 1992 client-based variables such as demographics, living conditions, diagnoses and mental health costs (for 1992 and 1991) to explain the variation in mental health and substance abuse costs.

Results: We find that the model that incorporates demographic characteristics, diagnostic information and cost data from two previous years explains about 16 percent of the in-sample variation and 10 percent of the out-of-sample variation in costs. A model that excludes prior cost covariates explains only 5 percent of the variation in costs. Despite the relatively low predictive power, we find some evidence that the disenrollment from the pilot capitation initiative input have been reduced if risk adjustment had been used to set capitation rates.
\end{abstract}

Discussion: The evidence suggests that even though risk adjustment techniques have room to improve, they are still likely to be useful

\footnotetext{
* Correspondence to: Kanika Kapur, RAND Corporation, 1700 Main Street,
} Santa Monica, CA 90401, USA

Contract grant sponsor: VISN 22 Mental Illness, Research, Education and Clinical Center of the Department of Veterans Affairs

Contract grant sponsor: NIMH UCLA-RAND Research Center on Managed Care for Psychiatric Disorders

Contract grant number: P50 MH-54623

Contract grant sponsor: Ernst Van Loben Sels Charitable Foundation

Contract grant sponsor: Zellerbach Family Fund for reducing risk selection in capitation programs. Blended payment schemes that combine risk adjustment with risk corridors or partial fee-for-service payments should be explored.

Implications for Health Care Provision, Use, and Policy: Our results suggest that risk adjustment methods, as developed to data, do not have the requisite predictive power to be used as the sole approach to adjusting capitation rates. Risk adjustment is informative and useful; however, payments to providers should not be fully capitated, and may need to involve some degree of risk sharing between providers and public mental health agencies. A blended contract design may further reduce incentives for risk selection by incorporating a partly risk-adjusted capitation payment, without relying completely on the accuracy of risk adjustment models.

Implications for Further Research: Risk adjustment models estimated using data sets containing better predictors of rehospitalization and more precise clinical information are likely to have higher predictive power. Further research should also focus on the effect of combination contract designs. Copyright (C) 2000 John Wiley \& Sons, Ltd.

Received 20 March 2000; accepted 4 September 2000

\section{Introduction}

Publicly funded mental health programs are increasingly implementing managed care systems, such as capitation, to control costs. Capitated contracts may result in disenrollment or adverse outcomes for individuals who have high costs and are severely mentally ill. ${ }^{1}$ Risk-adjusted payments to providers are likely to reduce providers' incentives to reject or under-treat clients who have high costs and are severely mentally ill. The literature on risk adjustment includes considerable research on Medicare and private populations. However, risk adjustment for individuals who are publicly funded and severely mentally ill has received far less attention. This study develops several risk adjustment models for this population and assesses their performance in predicting future costs.

A primary goal of risk adjustment is to align an individual's expected health cost with the prospective payment made to the individual's health care provider. This alignment reduces health care providers' incentives to select clients on the basis of their expected cost. ${ }^{2}$ Furthermore, risk adjustment may be necessary in order to preserve 
solvency in what can be relatively small private agencies.* Risk adjustment models use factors that are correlated with expected cost to set prospective payment rates. For example, demographic factors such as age, sex, race and insurance eligibility are commonly used. Risk adjustment models also use additional factors that are based on clinical information and past medical utilization.

This study uses Los Angeles County's Department of Mental Health administrative longitudinal data on clients who use mental health services from fiscal year (FY) 1991 to FY 1994 to develop and assess the performance of risk adjustment models for individuals with severe mental illness.* In this paper, we develop several models using data from FY 1991 to FY 1993. These models use clientbased variables such as demographics, living conditions, diagnoses and prior mental health costs to explain the variation in mental health and substance abuse costs. We apply the model with the highest explanatory power to predict costs for FY 1994, the first year of a Los Angeles County pilot capitation program for individuals with severe mental illness.

\section{Literature Review}

A great deal of research on risk adjustment has centered around the Health Care and Financing Administration's (HCFA) interest in using risk adjustment to set payment rates for total health care costs for its Medicaid and Medicare populations. $\dagger$ Since this research focuses on the general population and models total health costs, the results from this work may not be easily generalizable to risk adjustment for individuals with severe mental illness. However, the general findings from this research are an important guide to publicly financed mental health care research.

Past research has shown that demographic factors have limited explanatory power in risk adjustment models. ${ }^{4,5}$ Even with detailed diagnostic and lagged costs information, risk adjustment models explain less than 10 percent of variation. Newhouse et al. estimate that the maximum predictable share of variance for health costs is 14.5 percent. ${ }^{6}$ However, this estimate is calculated by relying on only time-invariant characteristics and, therefore, should be considered a lower bound on the true maximum predictable share of variance.

In a study of mental health and substance abuse risk adjustment, Ettner et al. assess the performance of several risk adjustment models under consideration by HCFA using a database of non-elderly employed individuals. ${ }^{5}$ None of the models explained more than 10 percent of the variation in costs, but the models that included comorbidities performed best. Ettner and Notman evaluated the predictive power of the ACG classification system, a set of diagnostic clusters

\footnotetext{
*In the event that a small community mental health agency, by chance, draws a high cost population, risk-adjusted capitation payments will enable the agency to receive higher capitation payments as long as the high costs for the population are reflected in prospective risk adjusters.

*In this paper, FY 1991 denotes FY 1990-1991 and so on.

$\dagger$ Greenwald et al. provide an overview of HCFA risk-adjustment research. ${ }^{3}$
}

and age and sex groupings using data on Medicaid enrollees in New Hampshire for FY 1993 and 1994. ${ }^{7}$ They examined total health care, mental health and substance abuse spending. For mental health and substance abuse spending, the maximum explanatory power obtained was $13 \%$ of the variance. Another study that developed risk adjustment outcome models for public mental health outpatient programs found that severe diagnoses, substance abuse and age were predictive of mental health outcomes such as functional status and health-related quality of life. ${ }^{8}$ A consistent finding in the research so far is that past diagnostic information has little predictive power in mental health costs. ${ }^{5}$

Newhouse has noted some challenges in developing risk adjustment models. ${ }^{2,9}$ One issue is that the use of certain adjusters may change provider behavior, reducing their effectiveness. For example, the use of prior inpatient costs as an adjuster may affect provider incentives to hospitalizean unintended consequence of the risk adjustment formula. On the other hand, ignoring past cost in the risk adjustment formula offers providers an incentive for risk selection. ${ }^{10}$ Furthermore, large databases are required to develop stable weights for risk adjustment models. However, the population with severe mental illness is relatively small, comprising only about 4 percent of the population, making it difficult to obtain large sample sizes. ${ }^{11}$ The high degree of variability in costs for this population also adds to the challenge in developing a model that explains a substantial share of the variation in costs.*

\section{Background: The Los Angeles PARTNERS Program}

In FY 1994, Los Angeles County Department of Mental Health (LACDMH) implemented the PARTNERS programa treatment program that used fixed annual per client payments to shift risk for treatment costs of high utilizers of public mental health services to private community-based treatment agencies. ${ }^{\dagger}$ PARTNERS was financed by moving funds from the support of 200 hospital beds to a new, intensive, capitated community-based treatment program for 500 high-cost clients. Six private, not-for-profit communitybased provider agencies were selected to participate in the program. Each was to provide care to either 50 or 100 selected clients. The agencies were paid at rates of between \$14 000 and \$21000 per client per year, depending on their bid. The capitation payments were not risk adjusted. In addition, these agencies were to be held financially responsible for treatment across all LACDMH institutional, crisis and outpatient settings, with the exception of pharmaceutical

\footnotetext{
*The difference between the 10th and the 90th percentile in the data is approximately $\$ 81000$.

$\dagger$ Young et al. provide a detailed description of the PARTNERS program implementation. ${ }^{13}$
} 
costs and acute hospitalizations paid for by fee-for-service Medicaid.*

Clients eligible for PARTNERS were chosen using LACDMH computerized administrative records and were restricted to a pool of individuals between 18 and 64 years old who had received treatment from LACDMH for at least three of the past five fiscal years, and who had been high utilizers of LACDMH fiscal resources. In order to be selected, a client's cost over the past five years had to average more than $\$ 30000$ per year, and, at the time of selection, be among the top $15 \%$ of all adult utilizers of LACDMH funded services. This average cost was based on those years when the client used LACDMH services. Cost data were not yet available for FY 1993, so assignment was based on services used between FY 1987 and FY 1992.

The community-based agencies were allowed to petition the mental health authority to disenroll clients who were unsuitable for community treatment. In addition, participation in the program was voluntary, so clients (or conservators on behalf) could refuse to participate. Clients who disenrolled from PARTNERS returned to usual LACDMH care. During the first year of the PARTNERS program, $76 \%$ of assigned clients were disenrolled. Previous work on the PARTNERS experience revealed two important findings. First, the economic incentives created by capitation contributed to, but were not fully responsible for, the disenrollment of PARTNERS clients. Second, analyses of health costs for enrollees in the PARTNERS capitation program suggest that the program did not result in a change in total costs. The program increased treatment costs for clients with lower pre-program costs, and decreased costs for the clients with high pre-program costs. The results suggested that future capitation programs for this severely ill population would benefit from using detailed clinical information to determine program eligibility and to set risk adjusted capitation rates. ${ }^{12,13}$

\section{Methods}

\section{Sample}

This analysis uses data provided by LACDMH for the years FY 1991 to FY 1994. The sample consists of clients who are eligible for the PARTNERS program; therefore, it comprises 'high utilizers' of public mental health funds.* To develop risk adjustment models, we use data from the

*For Medicaid patients, the portion of community-based organization costs that were Medicaid reimbursable were paid for the Medicaid program and that reimbursed payment was deducted from the capitation rate paid directly by LA County to the community organization. Hence, despite Medicaid reimbursement, the capitation feature of PARTNERS was preserved.

*By definition, the sample consists of repeat users of LACDMH services. According to LACDMH databases, 30 percent of clients in FY 2000 were new to the LACDMH system. The risk adjustment models developed here are not applicable to these new clients or to clients that are not severely mentally ill. period before PARTNERS implementation. ${ }^{\dagger}$ Risk adjustment models developed for all individuals who are publicly funded and mentally ill may not be appropriate for individuals who are costly and severely mentally ill; therefore, an advantage of this analysis is the focus on the sample of high utilizers.

\section{Data}

The data contain longitudinal information for all mental health service users on demographics, clinical status, utilization in public and private settings, and cost. The demographic data include date of birth, ethnicity, sex, type of residence, education level, marital status, language and living arrangements. Clinical information consists of variables such as type of treatment rendered (for example: acute hospital, state hospital, day treatment etc), length of treatment, Global Assessment Scale (GAS) ${ }^{16}$ DSM III-R diagnostic codes including secondary and dual diagnoses, private and public insurance information and Medicaid hospital and outpatient service use.* It is important to note that the client information in this data set is imperfect. For instance, diagnoses are often entered by the first clinician to see a patient and may not be updated to reflect current diagnoses until discharge diagnoses are entered. Other client variables such as GAS, homelessness and substance abuse may also fail to be updated. Costs in the database are in 1997 dollars-the costs were adjusted using the medical consumer price index. The sample used in this data set is restricted to individuals between the ages of 18 and 64 and has 1956 observations.

\section{Constructing the Analysis Variables}

Our primary goal is to estimate and predict annual costs for mental health and substance abuse services. Therefore, the outcome variable for the analysis is an annual measure of state and local mental health and substance abuse costs for FY 1993. It is important to note that the cost measure used in the analysis only captures costs of treatment borne by public mental health authorities, primarily LACDMH and Medicaid. Since costs incurred outside the LACDMH service areas are not captured in our data, our models should be interpreted as developing risk adjustment systems for public agencies. A public agency's success in contracting with outside agencies for services depends on its ability to predict costs.

Since Los Angeles County implemented the PARTNERS program in FY 1994, FY 1993 data were chosen to model risk adjustment. The analysis uses only one observation per

$\dagger$ The sample for the risk adjustment analysis has high costs during FY 1988-FY 1992. Since we estimate risk adjustment models for FY 1993 costs, a year that was not used to select high utilizers, we mitigate problems associated with modeling costs for a year that, by definition, is high cost. Since the population used in this estimation is severely and persistently mentally ill, it is unlikely that regression to the mean will bias the model estimates. This is substantiated by the relatively stable year to year correlation in costs presented in Table 2.

* Further details on this database are in Young et al. and Kapur et al. ${ }^{12,13}$ 
individual for the model rather than a panel data set with person-year observations since cost data from several lagged years are included in the risk adjustment model. In addition, the model chosen for analysis is unsuited for efficient estimation of person-year data sets.

Previous research has shown that rehospitalization, a large share of costs for individuals with severe mental illness, is associated with comorbid alcohol abuse, medication noncompliance and dangerous and aberrant behaviors. ${ }^{14,15}$ Therefore, we construct alcohol and drug abuse indicators, indicators for whether the client had been in jail, homeless and lagged outpatient costs to proxy for compliance.

Because this study aims to examine prospective risk adjustment, all explanatory variables are constructed using data from FY 1992.* All of the models include clientlevel demographic variables. We evaluate the following specifications. These specifications will be referred to by the abbreviations denoted in capital letters for the rest of the paper.

1. DEM. Includes only demographics—sex, age, Black, Hispanic, married, primary language is Spanish and primary language is neither Spanish nor English. $\dagger$

2. INS. Includes demographics, indicators for Medicaid insurance coverage, Medicare coverage and any other health insurance coverage. This model also adds an indicator for homelessness.

3. DIAG. Includes demographics, insurance indicators, homelessness, and indicators for diagnoses. The client's Global Assessment Scale (GAS) was also included in the model.

4. COST1. Includes demographics, insurance indicators, homelessness, diagnosis indicators and GAS. The following one-period lagged cost variables are also includedan indicator for any inpatient costs, an indicator for any outpatient costs, the logarithm of inpatient costs (set to zero if there are no lagged inpatient costs) and the logarithm of outpatient costs (set to zero if there are no lagged outpatient costs).

5. COST2. Includes demographics, insurance indicators, homelessness, diagnosis indicators, GAS, the four oneperiod lagged cost variables described in COST1, and the same four cost variables, lagged two periods.

To create the diagnosis indicators for DIAG, COST1 and COST2, we examined the primary and secondary diagnosis variables on all claims episodes for the year.* The following dichotomous diagnosis variables were created if either the

\footnotetext{
*Note that while we do use FY 1992 data, the diagnoses, GAS and homelessness variables may, in fact, not have been updated from the client's first contact with the county.

†While paying providers different amounts to treat patients of different races with otherwise comparable characteristics may be controversial, we included race variables in our models since their effects are interesting from a research perspective. Models without race variables had very similar predictive power and are available upon request. An indicator for 'Other race' was also included in some models; however this indicator was imprecisely estimated, and, therefore, dropped from the final models.

*If either the admission or the discharge diagnosis falls into the specified DSM III-R codes, the indicator for the appropriate diagnosis is turned on.
}

primary or secondary diagnosis fell into the following DSMIII-R categories: schizophrenia, bipolar disorder, psychotic disorder, major depression, alcohol or drug abuse, anxiety disorder, and dementia.*

For the sensitivity analysis, several additional variables and specifications were examined. First, the age variable was re-specified in six categories and interacted with sex. Second, lagged cost data was disaggregated into several components such as state hospital costs, case management costs, Institute for Mental Disease costs and Skilled Nursing Facility costs. Third, costs from the first and second lagged years were interacted and entered in the model to measure persistence of costs. Fourth, additional variables such whether or not the client had been in jail and prior living arrangements were also explored. Furthermore, interactions of diagnosis variables with sufficient cell sizes were also estimated. The results from these sensitivity analyses showed that these models were very similar in explanatory and predictive power to the model presented in detail in this paper.

\section{Analytic Approach}

Following a variant of the two-part model of Duan et al. that was proposed by Mullahy, we estimate a modified twopart model of mental health substance abuse costs: ${ }^{18,19}$

$$
\begin{aligned}
\operatorname{Pr}\left(\operatorname{cost}_{i t}>0\right) & =\alpha_{1}+\beta_{1} X_{i t-1}+\epsilon_{1 i t} \\
\text { Cost }_{i t} / \text { Cost }_{i t}>0 & =\exp \left(\alpha_{2}+\beta_{2} X_{i t-1}\right)+\epsilon_{2 i t}
\end{aligned}
$$

where $i$ indexes the client, $X$ is a vector of patient characteristics, $\beta$ is a vector of coefficients and $\epsilon$ is an error term. Equation (1) is a probit equation of probability that the client uses services in 1993. Equation (2) is a non-linear model of mental health and substance abuse costs, conditional on positive costs. Equation (2) is estimated using non-linear least squares. These two equations are suitable for modeling the relatively high proportion of non-utilizers and the skewness of costs.* Note that Mullahy's model differs from Duan's two-part model by specifying the second equation as a non-linear exponential model, rather than transforming the dependent variable to the logarithm of costs. ${ }^{18,19}$ This modified two-part model provided a better fit than the standard two-part model for these data. $\dagger$ The predictions

\footnotetext{
*An alternative specification, with clinically based hierarchical and mutually exclusive diagnoses, was also estimated. This specification yielded a slightly lower predictive power. The DSMIII-R codes are based on the classification provided by the American Psychiatric Association. ${ }^{17}$

*Two alternative models that respecified costs using the Box-Cox transformations that yielded the most symmetric distributions did not perform as well as the modified two-part model. The Box-Cox models were implemented as a single equation model for costs, and next, as a variant of the two part model, where the second equation of the model was specified using the Box-Cox transformation. After applying the appropriate variant of the smearing factor to retransform predicted costs to the original units, the mean predicted cost for the Box-Cox models was substantially lower than the actual mean in the data showing that these models did not fit the data very well. ${ }^{18}$

$†$ The modified two-part model was preferable to the standard two-part model in three ways: (1) the difference between the mean predicted expenditure and actual expenditure was smaller in the modified two-part model, (2) the error distribution from the second part of the modified twopart model was more symmetric than the distribution from the two-part model and (3) the modified two-part model yielded a higher $R^{2}$.
} 
from equation (1) and (2) enable the calculation of expected cost:

$$
E\left(\operatorname{Cost}_{i t}\right)=E\left[\operatorname{Pr}\left(\operatorname{Cost}_{i t}>0\right)\right] E\left(\text { Cost }_{i t} / \operatorname{Cost}_{i t}>0\right)
$$

\section{Results}

\section{Descriptive Statistics}

Table 1 reports descriptive statistics on all variables used in the estimation. $83 \%$ of the sample used mental health and substance abuse care and the average cost was \$25 294, substantiating that the individuals in our data are high cost. $79 \%$ of clients were eligible for Medicaid and 24\% were eligible for Medicare. $78 \%$ of the sample had schizophrenia, with smaller percentages having diagnoses of bipolar disorder, major depression and other psychoses. The data also show that, as expected, inpatient costs account for a large share of total costs.

It is also interesting to examine the extent of persistence

Table 1. Descriptive statistics for Los Angeles County data

\begin{tabular}{|c|c|}
\hline Variable & Mean \\
\hline Cost in $1993>0$ & $83 \%$ \\
\hline Cost in $1993(\$ 1)$ & $\begin{array}{c}25294 \\
(34612)\end{array}$ \\
\hline Age in 1992 & $\begin{array}{l}37 \\
(9)\end{array}$ \\
\hline Male & $57 \%$ \\
\hline Black & $34 \%$ \\
\hline Hispanic & $19 \%$ \\
\hline $\begin{array}{l}\text { Primary language is neither Spanish nor } \\
\text { English }\end{array}$ & $5 \%$ \\
\hline Primary language is Spanish & $6 \%$ \\
\hline Married in 1992 & $30 \%$ \\
\hline Medicaid coverage in 1992 & $79 \%$ \\
\hline Medicare coverage in 1992 & $24 \%$ \\
\hline Other insurance coverage in 1992 & $3 \%$ \\
\hline Homeless in 1992 & $15 \%$ \\
\hline Schizophrenia in 1992 & $78 \%$ \\
\hline Bipolar Disorder in 1992 & $28 \%$ \\
\hline Major Depression in 1992 & $12 \%$ \\
\hline Psychotic in 1992 & $37 \%$ \\
\hline Alcohol or Drug Abuse in 1992 & $18 \%$ \\
\hline Anxiety Disorder in 1992 & $3 \%$ \\
\hline Dementia in 1992 & $4 \%$ \\
\hline GAS in 1992 & $\begin{array}{l}34 \\
(10)\end{array}$ \\
\hline Outpatient costs in $1992(\$)$ & $\begin{array}{c}2755 \\
(4149)\end{array}$ \\
\hline Inpatient costs in $1992(\$)$ & $\begin{array}{c}22852 \\
(35114)\end{array}$ \\
\hline Outpatient costs in $1992>0$ & $62 \%$ \\
\hline Inpatient costs in $1992>0$ & $56 \%$ \\
\hline Outpatient costs in 1991 (\$) & $\begin{array}{c}2242 \\
(3766)\end{array}$ \\
\hline Inpatient costs in $1991(\$)$ & $\begin{array}{r}17068 \\
(31712)\end{array}$ \\
\hline Outpatient costs in $1991>0$ & $59 \%$ \\
\hline Inpatient costs in $1991>0$ & $43 \%$ \\
\hline Number of observations: 1956 & \\
\hline
\end{tabular}

Standard deviations provided in parentheses for continuous variables. in mental health and substance abuse costs over time for this sample of severely mentally ill. In Table 2, we present correlation coefficients for mental health and substance abuse expenditure, by type of expenditure and year. All costs exhibit a relatively stable correlation over time, as would be expected for this sample of severely mentally ill.* The correlation over time is somewhat smaller for outpatient costs than for inpatient and total costs.

\section{Predictive Ability of Risk Adjustment Models}

Several criteria are used to evaluate the predictive performance of the risk adjustment models. Table 3 summarizes the performance of the five risk adjustment models in predicting mental health and substance abuse costs.

In-Sample $R^{2}$ measures We use the Efron $R^{2}$, which accounts for bias as well as variance in the error, as a measure of the accuracy of prediction. This synthetic $R^{2}$ measure is defined as $1-\left[\Sigma\left(\mathrm{Util}_{i t}-E\left(\mathrm{Util}_{i t}\right)\right)^{2} / \Sigma\left(\mathrm{Util}_{i t}-\right.\right.$ $\left.\left.(1 / \mathrm{N}) \Sigma \mathrm{Util}_{i t}\right)^{2}\right]$. $\dagger$ Row 1 of Table 3 presents the Efron $R^{2}$. An additional measure, an adjusted $R^{2}$ is calculated by accounting for the number of explanatory variables used in the model. ${ }^{\ddagger}$ Row 2 presents the adjusted $R^{2}$ measure.

As expected, the basic demographics model, DEM,

Table 2. Correlation coefficients for health care expenditures, by type of expenditure and year

\begin{tabular}{lccccc}
\hline & $\begin{array}{c}\text { Cost in } \\
88\end{array}$ & $\begin{array}{c}\text { Cost in } \\
89\end{array}$ & $\begin{array}{c}\text { Cost in } \\
90\end{array}$ & $\begin{array}{c}\text { Cost in } \\
91\end{array}$ & $\begin{array}{c}\text { Cost in } \\
92\end{array}$ \\
\hline Total cost & & & & & \\
Cost in 88 & & & & & \\
Cost in 89 & 0.34 & & & & \\
Cost in 90 & 0.24 & 0.31 & & & \\
Cost in 91 & 0.18 & 0.27 & 0.36 & & \\
Cost in 92 & 0.15 & 0.18 & 0.22 & 0.32 & \\
Cost in 93 & 0.21 & 0.16 & 0.19 & 0.27 & 0.30 \\
Inpatient cost & & & & & \\
Cost in 88 & & & & & \\
Cost in 89 & 0.35 & & & & \\
Cost in 90 & 0.23 & 0.30 & & & \\
Cost in 91 & 0.18 & 0.27 & 0.37 & & \\
Cost in 92 & 0.16 & 0.17 & 0.22 & 0.33 & \\
Cost in 93 & 0.23 & 0.16 & 0.20 & 0.28 & 0.32 \\
Outpatient cost & & & & & \\
Cost in 88 & & & & & \\
Cost in 89 & 0.31 & & & & \\
Cost in 90 & 0.15 & 0.28 & & & \\
Cost in 91 & 0.14 & 0.17 & 0.33 & & \\
Cost in 92 & 0.08 & 0.13 & 0.23 & 0.29 & \\
Cost in 93 & 0.09 & 0.12 & 0.12 & 0.21 & 0.33 \\
\hline
\end{tabular}

*The year to year correlations decline slightly over time, but in general, they are relatively stable. This suggests that it is not necessary to model an auto-regressive error structure. ${ }^{6}$

$\dagger$ The Efron $R^{2}$ measure is used since the model in this paper is a nonlinear two-part model. ${ }^{20}$ Newhouse et al. and Ettner et al. also use the same $R^{2}$ measure..$^{5,6}$

$\dagger$ The adjusted $R^{2}$ is $1-\left((N+K)\left(1-R^{2}\right)\right) /(N-K)$, where $N$ is the number of observations and $K$ is the number of explanatory variables. Amemiya shows that this measure is equivalent to the 'prediction criterion'. ${ }^{21}$ 
Table 3. Predictive ability of modified two-part risk adjustment models

\begin{tabular}{lrrrrrr}
\hline \multicolumn{1}{r}{ Model name } & DEM & INS & DIAG & COST1 COST2 \\
\hline (1) Efron $R^{2}$ & 0.006 & 0.012 & 0.049 & 0.137 & 0.164 \\
(2) Adjusted $R^{2}$ & -0.002 & -0.001 & 0.029 & 0.116 & 0.140 \\
(3) Out-of-sample $R^{2}$ & -0.003 & 0.009 & 0.027 & 0.087 & 0.103 \\
Predictive & & & & & \\
ratios & & & & & \\
(4) Full sample & 1.000 & 1.000 & 1.000 & 0.990 & 0.992 \\
(5) Male, under 45 & 1.008 & 1.017 & 1.012 & 1.021 & 1.018 \\
(6) Female, under 45 & 1.002 & 0.993 & 1.007 & 0.972 & 0.989 \\
(7) Male, 45-64 & 0.954 & 0.945 & 0.928 & 0.946 & 0.928 \\
(8) Female, 45-64 & 0.993 & 0.981 & 0.986 & 0.920 & 0.922 \\
(9) Schizophrenia & 0.927 & 0.928 & 1.001 & 0.989 & 0.991 \\
(10) Bipolar Disorder & 0.980 & 0.974 & 1.020 & 0.989 & 1.003 \\
(11) Psychotic & 0.899 & 0.910 & 1.011 & 0.980 & 0.993 \\
(12) Major Depression & 1.326 & 1.328 & 1.037 & 1.085 & 1.085 \\
\hline
\end{tabular}

Notes:

(i) The model definitions are: DEM, demographics only; INS, DEM + insurance + homelessness; DIAG, INS + diagnoses + GAF score; COST1, DIAG +1 year lagged cost; COST2, COST1 + 2 year Lagged cost.

(ii) The maximum $R^{2}$ is .345 for these data (calculated using data from FY 1988 to FY 1993).

(iii) Predictive ratios are the ratio of predicted to actual FY 1993 costs.

performs the worst, with an $R^{2}$ of less than 1 percent, and a negative adjusted $R^{2} *$ Adding insurance indicators and homelessness in INS increases the $R^{2}$ to over 1 percent. The model that performs the best, with an $R^{2}$ of 16 percent, is COST2 that includes costs variables from 1992 and 1991. Note that COST2 only outperforms COST1, but COST2 has far greater data demands, requiring two lagged periods of cost data. Both COST1 and COST2 have much higher $R^{2}$ than the models that include no lagged cost data. $\dagger$

Out-of-Sample $R^{2}$ measures An important criterion for evaluating the predictive ability of a prospective-risk adjustment model is its performance in projecting out-of-sample costs. Since the sample used to develop the risk adjustment models is relatively small, there is a potential for overfitting the data; however, out-of-sample predictive ability is less influenced by an over-fitted model. To evaluate the performance of the risk adjustment models, we use the parameter estimates obtained from the model of FY 1993 costs and apply these estimates to FY 1993 client characteristics to predict FY 1994 costs. Next, we compare FY 1994 predicted costs to actual FY 1994 costs using the $R^{2}$ measure described above. A problem with this out-ofsample predictive ability check is that it relies on FY 1994 cost data. As described earlier, Los Angeles County

*The formula for the adjusted $R^{2}$ is such that it can be negative when the $R^{2}$ is cost close to zero. The adjusted $R^{2}$ is $1-\left((N+K)\left(1-R^{2}\right)\right) /(N-K)$, where $N$ is the number of observations and $K$ is the number of explanatory variables.

$\uparrow$ We also calculate the mean absolute prediction error that averages the absolute value of the difference between the realized cost and the model prediction over the sample. We find that this measure yields the same ranking of model performance as the $R^{2}$ measure reported in Table 3 . Unlike the $R^{2}$ measure and predictive ratios, the mean absolute prediction error is not standardized and therefore, is harder to interpret. Therefore, we report the $R^{2}$ and predictive ratios in the text. implemented a capitation program in FY 1994; therefore, costs reported from FY 1994 on are likely to be influenced by the capitation program's cost control or service reporting incentives.* We find the out-of-sample $R^{2}$, reported on row 3 of Table 3, is somewhat lower than the in-sample $R^{2}$. It is important to note that the out-of-sample $R^{2}$ yields the same ranking of model performance as the $R^{2}$.

Maximum $R^{2}$ Using the methodology proposed in Newhouse et al., we calculate the maximum $R^{2}$ using data from FY 1988 to FY 1993 as 0.345 . $†$ The maximum $R^{2}$ provides an indication of the maximum predictable share of variance for these data. Since this estimate is calculated by relying on only time-invariant characteristics, it should be considered a lower bound on the true maximum predictable share of variance. As a share of the maximum $R^{2}$, the model with the highest predictive power (COST2) explains less than half of the predictable variance.

Predictive Ratios Finally, we calculate predictive ratios for the risk adjustment models. Predictive ratios allow a comparison of the financial incentives that community agencies face in enrolling different client subgroups. The predictive ratio is calculated as the total predicted FY 1993 cost for a group divided by the actual FY 1993 cost for the same group. A model performs well for a subgroup when its predictive ratio is close to one. ${ }^{4} \ddagger$ Rows 4 to 12 report predictive ratios for the five risk adjustment models for the full sample and selected sub-samples. As expected, the models DIAG, COST1 and COST2 that include diagnoses have predictive ratios that are close to one for the diagnosis sub-group samples. However, these models perform relatively worse than the simpler models on the demographic subgroup samples.

\section{Model Estimates}

In Table 4, we present the estimates from the COST2 specification.* In column 2, the marginal effects from the probit equation show that no demographic factors have statistically significant impacts on the probability of use. A diagnosis of anxiety disorder raises the probability of having positive mental health costs by 13 percentage points (relative to a base probability of use of 83 percent). The coefficients

\footnotetext{
* Since part of our sample was randomly assigned to the program, we could use the part of the sample that was not assigned to the program in FY 1994 to calculate the out-of-sample $R^{2}$. However, due to high disenrollment from PARTNERS for high cost enrollees, and subsequent assignment of the high cost 'control group', the sub-sample that is not assigned to PARTNERS is a relatively low cost population that is quite different from the sample that was used to develop the risk adjustment models. Therefore, we do not use this sub-sample to calculate out-ofsample predictive power.

$\dagger$ Newhouse et al. calculate a maximum $R^{2}$ of.145 for their health expenditure data, using shorter panels of 3 to 5 years. This statistic is obtained as the $R^{2}$ of a model with a full set of client specific indicator variables. ${ }^{6}$

* Since the models estimated in this paper are non-linear, the predictive ratio for the full sample may not necessarily be equal to one.

*Estimates from DIAG, the model that excludes prior cost variables, have a similar pattern, but are somewhat larger in absolute value than the
} estimates from COST2. These estimates are available upon request. 
Table 4. Modified two-part model estimates for FY 1993 (specification: cost2)

\begin{tabular}{|c|c|c|c|c|}
\hline \multirow[b]{2}{*}{ Variables } & \multicolumn{2}{|c|}{$\begin{array}{l}\text { Equation (1) } \\
\text { probit model }\end{array}$} & \multicolumn{2}{|c|}{$\begin{array}{c}\text { Equation (2): } \\
\text { exponential model }\end{array}$} \\
\hline & $\begin{array}{c}\text { Marginal } \\
\text { effect }\end{array}$ & $\begin{array}{l}\text { Standard } \\
\text { error }\end{array}$ & Coefficient & $\begin{array}{l}\text { Standard } \\
t \text { error }\end{array}$ \\
\hline \multicolumn{5}{|l|}{ Demographic variables } \\
\hline Age in 1992 & -0.001 & 0.001 & $-0.006^{*}$ & 0.003 \\
\hline Male & 0.001 & 0.018 & 0.038 & 0.053 \\
\hline Black & 0.012 & 0.019 & -0.026 & 0.056 \\
\hline Hispanic & 0.008 & 0.026 & -0.094 & 0.081 \\
\hline $\begin{array}{l}\text { Primary language is } \\
\text { neither Spanish nor }\end{array}$ & & & & \\
\hline $\begin{array}{l}\text { English } \\
\text { Primary language is }\end{array}$ & -0.004 & 0.038 & -0.042 & 0.112 \\
\hline Spanish & -0.009 & 0.040 & 0.114 & 0.104 \\
\hline Married in 1992 & -0.010 & 0.020 & -0.073 & 0.063 \\
\hline Insurance variables & & & & \\
\hline $\begin{array}{l}\text { Medicaid coverage in } \\
1992\end{array}$ & 0.023 & 0.021 & $-0.150 *$ & 0.055 \\
\hline $\begin{array}{l}\text { Medicare coverage in } \\
1992\end{array}$ & 0.018 & 0.019 & $-0.115^{*}$ & 0.063 \\
\hline $\begin{array}{l}\text { Other insurance coverage } \\
\text { in } 1992\end{array}$ & -0.053 & 0.054 & -0.237 & 0.166 \\
\hline $\begin{array}{l}\text { Homeless in } 1992 \\
\text { Diagnoses and } \\
\text { functioning variables }\end{array}$ & -0.020 & 0.026 & 0.083 & 0.066 \\
\hline Schizophrenia in 1992 & 0.033 & 0.023 & $0.252 *$ & 0.085 \\
\hline Bipolar Disorder in 1992 & -0.012 & 0.021 & $0.104 *$ & 0.059 \\
\hline $\begin{array}{l}\text { Major Depression in } \\
1992\end{array}$ & -0.015 & 0.028 & -0.038 & 0.096 \\
\hline Psychotic in 1992 & 0.006 & 0.019 & $0.116^{*}$ & 0.057 \\
\hline $\begin{array}{l}\text { Alcohol or Drug Abuse } \\
\text { in } 1992\end{array}$ & 0.024 & 0.022 & 0.077 & 0.062 \\
\hline Anxiety Disorder in 1992 & $20.125^{*}$ & 0.028 & $0.445^{*}$ & 0.111 \\
\hline Dementia in 1992 & -0.019 & 0.045 & $0.237 *$ & 0.101 \\
\hline GAS in 1992 & 0.000 & 0.001 & $-0.008 *$ & 0.003 \\
\hline $\begin{array}{l}\text { Prior cost variables } \\
\text { Outpatient costs in } 1992\end{array}$ & 0.015 & 0.010 & -0.045 & 0.035 \\
\hline $\begin{array}{l}\text { Inpatient costs in } 1992 \\
\text { (in log. \$) }\end{array}$ & 0.004 & 0.010 & $0.355^{*}$ & 0.038 \\
\hline $\begin{array}{l}\text { Outpatient costs in } \\
1992>0\end{array}$ & -0.077 & 0.075 & 0.156 & 0.266 \\
\hline $\begin{array}{l}\text { Inpatient costs in } \\
1992>0\end{array}$ & 0.002 & 0.098 & $-3.544^{*}$ & 0.429 \\
\hline $\begin{array}{l}\text { Outpatient costs in } 1991 \\
\text { (in log. \$) }\end{array}$ & 0.012 & 0.011 & 0.042 & 0.034 \\
\hline $\begin{array}{l}\text { Inpatient costs in } 1991 \\
\text { (in log. \$) }\end{array}$ & $0.027 *$ & 0.009 & $0.115^{*}$ & 0.029 \\
\hline $\begin{array}{l}\text { Outpatient costs in } \\
1991>0\end{array}$ & -0.058 & 0.079 & $-0.575^{*}$ & 0.271 \\
\hline $\begin{array}{l}\text { Inpatient costs in } \\
1991>0 \\
\text { Number of observations: } \\
1956\end{array}$ & $-0.229 *$ & 0.104 & $-0.980 *$ & 0.316 \\
\hline
\end{tabular}

Note: Marginal effects for equation (2) can be obtained by multiplying the coefficients by $\$ 25085$, the mean prediction for this model. *denotes significance at the $5 \%$ level. on the lagged inpatient cost variables for FY 1991 suggest that clients with high prior inpatient use tend to be more likely to use mental health services. $\dagger$ Column 4 shows the coefficients from the second equation. Note that multiplying the coefficients by $\$ 25085$ gives the approximate marginal effects. The results show that age has a relatively small negative effect on costs - an extra year decreases costs by about $\$ 150$. Note that this result should be interpreted in conjunction with the fact that the sample contains clients between the ages of 18 and 64; furthermore, $90 \%$ of the sample is younger than age 50. Clients with Medicaid coverage use approximately $\$ 3700$ less in services. Schizophrenia appears to increase costs by approximately $\$ 6300$. Improved functioning as measured by the GAS statistically significantly reduces costs. The coefficients on the lagged inpatient cost variables suggest that clients with high inpatient use tend to be persistently high mental health users. * The impact of lagged outpatient cost is smaller and somewhat mixed.

\section{An Application: Risk Adjustment of a Los Angeles Capitation Program}

As described earlier, in FY 1994 LA County implemented a capitation initiative called PARTNERS that was subject to high disenrollment. In this section, we ask the following question: is there evidence that risk adjustment methods could have reduced disenrollment from PARTNERS? To address this question, we determine whether the clients who were disenrolled in FY 1994 had the largest difference between their projected risk adjusted costs for FY 1994 and their capitation payment rate. Community treatment agencies would have had the greatest incentive to disenroll those clients for whom the expected cost for FY 1994 (estimated using risk adjustment) exceeded the capitation payment. Our measure of disenrollment includes all separations from PARTNERS agencies since data on the reason for disenrollment may be problematic. $\dagger$

We use estimates obtained in the previous section from the COST2 model for FY 1993 costs-note that the risk adjustment model was estimated on data from the year

\footnotetext{
$\dagger$ The combination of the negative effect of the indicator for past inpatient use and the positive effect of the logarithm for past inpatient use implies that clients with FY 1991 inpatient costs of \$5000 are more likely to incur mental health costs in FY 1993.

*The combination of the negative effect of the indicator for past inpatient use and the positive effect of the logarithm for past inpatient use implies that clients with FY 1991 inpatient costs of $\$ 4000$ and more have higher costs in FY 1993. Note that the distribution of inpatient costs is extremely skewed-about half the sample has no inpatient costs and the other half of the sample has mean inpatient costs of approximately $\$ 40000$.

$\dagger$ While we have data on the reason for disenrollment, the criteria for disenrollment were developed during the implementation of the program and may have been somewhat malleable. Despite these problems, a multinomial logit model of disenrollment criteria is estimated. The results show that the impact of costs and other key factors on the relative risks of being disenrolled are quite similar between the disenrollment categories. This supports the conjecture that these categories were malleable.
} 
preceding PARTNERS implementation.* We apply the parameter estimates obtained from the model of FY 1993 costs to FY 1993 client characteristics to predict FY 1994 costs. Assuming that the PARTNERS agencies' expectation of FY 1994 costs are aligned with the predicted FY 1994 costs from the risk adjustment model, the probability of disenrollment from PARTNERS is likely to increase as the gap between the FY 1994 predicted cost and the FY 1994 fixed capitation payment increases. As expected, we find a positive correlation of 0.20 between the probability of being disenrolled and the difference between the FY 1994 risk adjusted cost obtained from our model and the fixed capitation rate paid to the PARTNERS agencies.

Given that risk adjustment methods predict a relatively small share of future costs, Newhouse and others have endorsed the use of blended payment schemes, where prospective capitation payments are combined with reimbursement for actual costs. ${ }^{2,9}$ The prospective capitation component of the blended payment to community mental health providers would preserve providers' incentives to utilize resources efficiently, whereas the cost reimbursement component of the blended payment would reduce providers' exposure to risk.

As an illustrative example, we explore the projected impact of two types of blended payment scheme on the PARTNERS disenrollment problem. In this example, we use the predictions from the risk adjustment model as a proxy for community agencies' expectations of enrollee costs. First, we examine supplementing the existing PARTNERS capitation payments that are not risk adjusted with a risk corridor payment scheme. In this payment scheme, if the difference between the realized cost and the capitation payment exceeds the 75 th percentile of the distribution, the county would pay the community agency 80 percent of the difference between the realized cost and the 75th percentile. Similarly, if the difference between the realized cost and the capitation payment falls below the 25th percentile, the county would collect 80 percent of the difference between the 25 th percentile and the realized cost from the community agency. Applying this payment scheme to the existing distribution of expected FY 1994 costs (as predicted by the risk adjustment model) and disenrollment, we calculate that the disenrollment rate for high cost clients (with expected FY 1994 costs over the 95th percentile) would fall from 92 percent to 80 percent.* The overall disenrollment rate would

\footnotetext{
*Using a model with lagged costs as covariates to set risk adjustment rates on an ongoing basis may be inappropriate since this model would blunt the incentives of capitation. However, in this analysis, we are using such a model only to analyze rates for the first year of a capitation program. *To implement this example, we assume that providers classify clients according to three cost tiers-95th percentile and over, 50th to 95th percentile and under the 50th percentile. The disenrollment rate for each tier is the average disenrollment rate for all clients within that tier in the sample. The data support this three-tier cost classification. While it is possible to estimate a multivariate model of disenrollment on costs predicted using the risk adjustment model and other covariates, this model would be identified only on the basis of functional form. Furthermore, given small sample sizes, there is a serious concern about over-fitting the data with this model. Therefore, we rely on a non-parametric analysis of risk adjustment and disenrollment.
}

fall from 69.4 percent to 68.6 percent. The second example examines the impact of combining PARTNERS capitation payments with a 'coinsurance'- the county pays half of providers' actual costs plus half of the PARTNERS capitation payment. As with the risk corridor, the coinsurance arrangement reduces disenrollment for high cost clients to 81 percent and overall disenrollment by less than 1 percentage point. Blended payment schemes appear to have a small effect on reducing disenrollment for PARTNERS clients. It is important to note that PARTNERS was associated with a high disenrollment rate even for relatively low cost clients. Therefore, changing payment incentives for high cost clients cannot be expected to greatly reduce disenrollment.

\section{Conclusion and Discussion}

This study uses administrative longitudinal data on mental health users from FY 1991 to FY 1994 to develop and assess the performance of risk adjustment models for individuals with severe mental illness. Using FY 1992 characteristics to predict FY 1993 costs, we find that the model that incorporates demographic characteristics, diagnostic information, and cost data from two previous years explains only about 16 percent of the in-sample variation in costs and 10 percent of the out-of-sample variation in costs. Furthermore, a model excluding prior cost covariates explains only about 5 percent of the variation in costs.

Using the risk adjustment model developed in this paper, we estimate costs for the first year of a pilot capitation program that was subject to a high rate of disenrollment. We find some evidence that the disenrollment from this capitation initiative may have been reduced if risk adjustment had been used to set capitation rates; however, the effects of blended payment schemes on disenrollment appear to be small.

This study has several limitations; therefore, it important to interpret the results cautiously. First, this study focuses on developing a risk adjustment system specifically for individuals who are publicly funded and severely mentally ill. The results are unlikely to generalize to lower cost populations. Second, the study is limited by the accuracy of the data. Clinical information for the clients was not verified. Furthermore, the database did not include variables that may be useful in predicting rehospitalization. Risk adjustment models estimated using richer data sets containing predictors of rehospitalization and better clinical information are likely to have higher predictive power. In addition, due to our focus on individuals with severe mental illness, we have a relatively small sample size that reduces the precision of the estimates. The ability of county mental health authorities to successfully risk adjust payments in future will depend on the development of relevant and detailed data systems. Third, as described earlier, this study only models costs incurred within the LACDMH system. Costs incurred in other counties are not measured in this database. Lastly, since the risk adjustment models are estimated on a sample of clients who are persistently and severely mentally 
ill, we may have expected a predictive power higher than 16 percent. In the models presented in this paper, prior inpatient costs appeared to have the highest predictive power-clients with high inpatient costs tended to be persistently high mental health users. However, as noted by Newhouse, using prior inpatient cost as an adjuster may affect providers' incentives to hospitalize - an undesirable consequence. ${ }^{2}$ Consistent with the literature, diagnoses had relatively little predictive power. In the model using no prior cost data, predictive power was only about 5 percent. In this sample, this is most likely due to the fact that 78 percent of the sample had schizophrenia; therefore, diagnosis data at this level of precision is a relatively blunt adjuster. Furthermore, the relationship between diagnosis and severity is weak in mental health settings.

Our results suggest that risk adjustment methods, as developed to date, do not have the requisite predictive power to be used as the sole measure in setting capitation rates. These results are particularly interesting since states such as California are considering the implementation of full capitation for counties. Certainly, risk adjustment is informative and useful, as shown in the application to the Los Angeles county capitation program; however, as suggested by Newhouse ${ }^{2,9}$ and endorsed by Ettner et al., payments to providers should not be fully capitated, but rather should involve some degree of risk sharing between providers and public mental health agencies. This combination contract design may reduce incentives for risk selection by incorporating a partly risk adjusted capitation payment, without relying completely on the accuracy of risk adjustment models.

\section{Acknowledgements}

This research was supported by the VISN 22 Mental Illness, Research, Education and Clinical Center of the Department of Veterans Affairs, by the NIMH UCLA-RAND Research Center on Managed Care for Psychiatric Disorders (grant P50 MH-54623), by the Ernst Van Loben Sels Charitable Foundation and by the Zellerbach Family Fund. The authors thank Audrey Burnam, Areta Crowell, Paul Devereux, London Fairborn, Ron Florendo, Emmett Keeler, Paul Koegel, Rod Shaner, Marvin Southard, Greer Sullivan and two anonymous referees. The opinions expressed in this article are only the authors'.

\section{References}

1. Young AS, Sullivan G, Burnam AM et al. Measuring the quality of outpatient treatment for schizophrenia. Arch Gen Psychiatry 1998; 55: 611-617.

2. Newhouse JP. Risk adjustment: where are we now? Inquiry 1998; 35: $122-131$.

3. Greenwald LM, Esposito A, Ingber MJ et al. Risk adjustment for the Medicare program: lessons learned from research and demonstrations. Inquiry 1998; 35: 193-209.

4. Ellis RP, Pope GC, Iezzoni LI et al. Diagnosis-based risk adjustment for Medicare capitation payments. Health Care Financing Rev 1996; 17 (3): 101-128

5. Ettner SL, Frank RG, McGuire TG et al. Risk adjustment of mental health and substance abuse payments. Inquiry 1998; 35: 223-239.

6. Newhouse JP, Manning WG, Keeler EB et al. Adjusting capitation rates using objective health measures and prior utilization. Health Care Financing Rev 1989; 10 (3): 41-54.

7. Ettner SL, Notman EH. How well do ambulatory care groups predict expenditures of mental health and substance abuse patients? J Admin Policy Mental Health 1997; 24 (4): 339-358.

8. Hendryx MS, Dyck DG, Srebnik D. Risk-adjusted outcome models for public mental health outpatient programs. Health Services Res 1999; 34 (1): 171-195.

9. Newhouse JP. Patients at risk: health reform and risk adjustment. Health Affairs 1994; 13 (1): 132-146.

10. Van Vliet R, van de Ven W. Towards a capitation formula for competing health insurers: an empirical analysis. Soc Sci Med 1992; 34(9): 1935-1048.

11. Frank RG, McGuire TG. Economics and Mental Health, NBER Working Paper 7052. Cambridge, MA, 1999

12. Kapur K, Young AS, Murata D, et al. The economic impact of capitated care for high utilizers of public mental health services: The Los Angeles PARTNERS Program Experience. J Behav Health Services Res 1999; 26 (4): 415-428.

13. Young AS, Sullivan G, Murata D, et al. Implementing publicly funded risk contracts with community mental health organizations. Psychiatric Services 1998; 49 (12): 1579-1584.

14. Sullivan G, Young AS, Morgenstern H. Behaviors as risk factors for rehospitalization: implications for predicting and preventing admissions among the seriously mentally ill. Soc Psychiatry Psychiatr Epidemiol 1997; 32: 185-190.

15. Sullivan G, Wells KB, Morgenstern H, Leake B. Identifying modifiable risk factors for rehospitalization: a case-control study of seriously mentally ill persons in Mississippi. Am J Psychiatry 1995; 152: 1749-56.

16. Jones SH, Thornicroft G, Coffey M, Dunn G. A brief mental health outcome scale-reliability and validity of the Global Assessment of Functioning (GAF). Br J Psychiatry 1995; 166: 654-9.

17. American Psychiatric Association. Diagnostic and Statistical Manual of Mental Disorders: DSM-IIIR. Washington, DC, 1994.

18. Duan, N, Manning, WG, Morris C et al. A comparison of alternative models for the demand for medical care. J Business Econ Stat 1983; 1 (2): 115-126.

19. Mullahy J. Much ado about two: reconsidering retransformation and the two-part model in health econometrics. J Health Econ 1998; 17 (3): 247-382.

20. Efron B. Regression and ANOVA with zero-one data: measures of residual variation. J Am Stat Assoc 1978; 73 (1): 113-121.

21. Amemiya T. Advanced Econometrics. Harvard University Press: Cambridge, MA, 1985. 